Original Research Paper

\title{
Enhancing the Performance of Wireless Routing Protocols of MANET using AI
}

\author{
Yahia Hasan Jazyah \\ Department of ITC, Arab Open University, Kuwait
}

Article history

Received: 15-04-2021

Revised: 26-08-2021

Accepted: 08-09-2021

Email: yahia@aou.edu.kw

\begin{abstract}
The main goal of Mobile Ad hoc Network (MANET) is connecting mobile devices directly without central point (peer to peer), which makes it popular area of research. MANET is running using routing protocols that enable mobile devices (nodes) to communicate to each other. Different types of routing protocols have been designed that depend on different strategies in order to establish and maintain connections between mobile nodes. Nowadays, Artificial Intelligence (AI) has involved in all fields of science and technology aiming to enhance the performance and gaining accurate results. This research aims to design and implement a wireless routing protocol for MANET based on AI (Ant-OBS - Ant on Behalf of Source) in order to enhance the performance of network in terms of network overhead and power consumption. The proposed protocol benefits from the behavior of ant insect. A mathematical model is designed to model the proposed protocol; the output of the model performs well when it is compared to AODV and LAR1 routing protocols.
\end{abstract}

Keywords: MANET, Routing Protocol, AI, AODV, LAR1

\section{Introduction}

Wireless Local Area Networks (WLAN) enables nodes to access each other within intra-network or internetwork. In some cases, communicating nodes are not in the same transmission range and so intermediate nodes are required to establish and maintain connection between source and destination in a multichip route mode under the control of wireless routing protocol. Wireless routing protocols that provide this key functionality are classified as topological or position based routing protocols. (Corson and Macker, 1999).

\section{A) Topological Based Routing Protocols}

Topology based routing protocols use existing information about the network to flood (or forward) packets. There are two main routing strategies which are classified as topology based and these are: Proactive and reactive routing protocols. In addition to hybrid protocols which mix the previous two types. (Mauve et al., 2001)

\section{a) Proactive Routing Protocols}

Proactive (or table driven) protocols (Mauve et al., 2001) maintain routing information for each node in the network and store information in routing tables. This information is then updated whenever the topology changes and so one or more routing tables are required by each node to store routing information. Most proactive strategies share the same features, but they differ in the number of routing tables and frequency of topological update. Examples of proactive routing protocols are Destination-Sequenced Distance Vector (DSDV) (Abolhasan et al., 2004), Cluster-head Gateway Switch Routing (CGSR) (Abolhasan et al., 2004), Wireless Routing Protocol (WRP) (Jazyah and Hope, 2010) and Optimized Link State Routing Protocol (OLSR) (Jacquet et al., 2001).

\section{b) Reactive Routing Protocols}

Reactive routing protocols maintain route information on demand, i.e., when source node wants to send message to destination, it initiates Route Request (RREQ) to find route to destination. When a route fails, a route maintenance process is launched to repair the failed route.

Ad hoc On-Demand Distance Vector (AODV) (Perkins et al., 2003), Dynamic Source Routing (DSR) (Johnson et al., 2001), Temporally Ordered Routing Algorithm (TORA) (Jazyah and Hope, 2010) and Associativity-Based Routing (ABR) (Abolhasan et al., 2004) are examples of reactive strategy. 


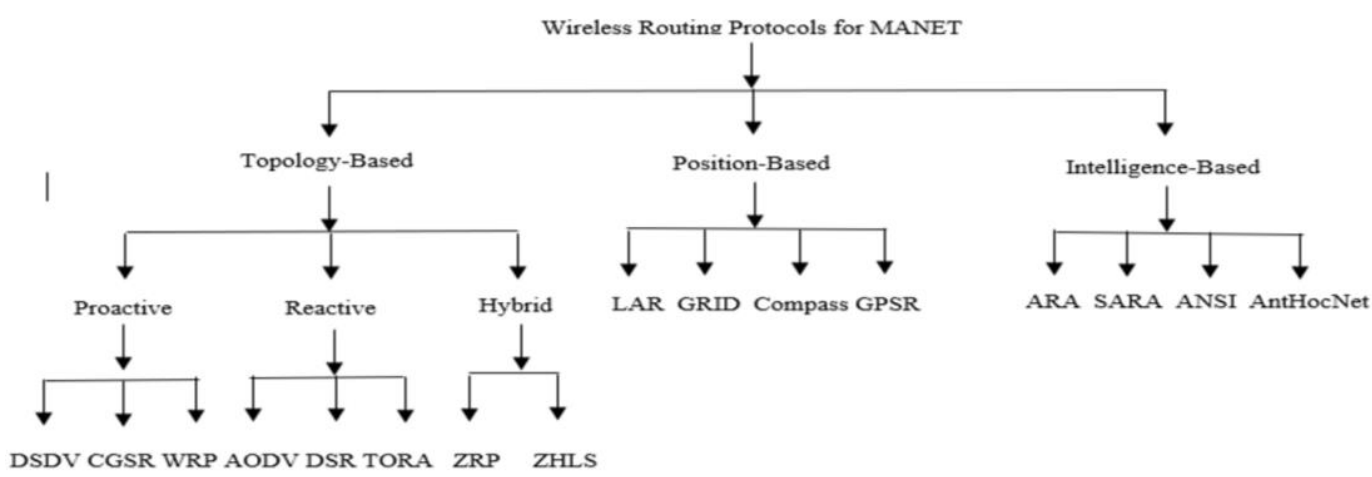

Fig. 1: Categorization of Ad hoc routing protocols

\section{c) Hybrid Routing Protocols}

Most networks are partitioned into zones (or zonebased) or nodes are grouped into trees or clusters. Hybrid routing protocols merge proactive and reactive strategies whereas proactive strategy is applied within zone (or intra zone), while a reactive strategy is applied outside zone (or inter zone). The following are examples of hybrid routing protocols: Zone Routing Protocol (ZRP) (Beijar, 2002) and Zone-based Hierarchical Link State (ZHLS) (Abolhasan et al., 2004).

\section{B) Position Based Routing Protocols}

This type of protocols enables source node to flood packets towards destination using positional information based on previous knowledge about its location, which reflects positively on network overhead and power consumption, such as Location Aided Routing Protocol (LAR) (Ko and Vaidya, 2000), GRID (Liao et al., 2000), Compass (Kranakis et al., 1999) and Greedy Perimeter Stateless Routing (GPSR) (Karp and Kung, 2000).

\section{C) Intelligence Based Protocols}

A current classification of routing protocols based on AI is the intelligence-based routing protocols. They are now encouraging researchers for more investigation. This type of routing protocols is based on the behavior of animals that form swarms. Two popular groups of such protocols are beeand ant-inspired protocols, which take their principles from ants and bees' colonies (Manibushan et al., 2016), Fig. 1.

This study proposes routing protocol for MANET based on ant's behavior. The aim of proposed protocol is to reduce the power consumption and network overhead by confining the propagation of Route Request (RREQ) from the source towards the destination node.

A mathematical model is designed to prove the correctness of the proposed Protocol-Ant on Behalf Source (Ant-OBS). Numerical values are obtained from the model for both the Ant-OBS and LAR1 routing protocols in order to justify the performance of the proposed one. The result shows that Ant-OBS outperforms AODV and LAR1.

\section{Related Work and Motivation}

The proposed routing protocol exploits the advantages of LAR scheme 1 (LAR1) and intelligencebased routing protocols.

\section{A) Location Aided Routing Protocol (LARz}

LAR (Ko and Vaidya, 2000) is an on-demand routing protocol that uses the modified Dijkstra's Algorithm to find the shortest path. Destination lies in the center of a circular region of certain radius at a certain time known as Expected Zone, as shown in Fig. 2, which indicates which zone of the network should be reached by RREQ. GPS enables terminals to know its own position and speed, while dissemination is performed by piggybacking location information in all routing packets. "Piggybacking is a bi-directional data transmission technique in the network layer. It makes the most of the sent data frames from receiver to emitter and adds the confirmation that the data frame sent by the sender was received successfully acknowledge" (Ucan e. al., 2007). LAR1 defines request zone that includes sender and receiver on opposite corner of a rectangle. Rectangle dimensions are estimated according to the receiver average speed at a certain time. Only nodes within the zone respond to the RREQ of sender. LAR1 reduces network overhead, but causes delay when route is found. Different shapes can be used other than the rectangle such as circle, cone and bar.

\section{B) Ad hoc On-Demand Distance Vector (AODV)}

AODV (Perkins et al., 2003) is a Distance Vector (DV) routing protocols; where every node knows about its neighbors and the costs incurred in order to reach them using the Bellman-Ford algorithm (Faychuk et al., 2019).

AODV is a reactive shortest single path wireless routing protocol based on DSDV protocol. When a source wants to send a message to a destination, it checks its routing table, if there is a valid route to destination, it starts sending packets immediately. If not, it broadcasts 
RREQ to all neighbor nodes. It should be noted that RREQ contains the fields: Hop count, source and destination sequence numbers, destination and source addresses, RREQ ID and other pre-determined fields. When an intermediate node receives a RREQ, it checks its routing table for a path to the destination, if it exists, it unicasts a route reply (RREP) to the source, otherwise, it increases the hop count by one and adds its ID to the RREQ and then re-broadcasts it to its neighbor's; the process continues until the RREQ reaches its destination. The destination then selects the first coming RREQ and unicasts RREP using the reverse path to source node. When the source receives several RREPs, it selects the route of the highest sequence number and minimum hop count and then it establishes the route and starts sending packets.

To guarantee loop freedom, the source node uses a sequence number and includes it in RREQ. When a node receives a control message (RREQ, RREP, or RERR), it checks its routing table for an entry to the specified destination, if there is no entry in the routing table about the destination, it creates a new one. If there is an entry in the routing table, the route is only updated if the new sequence number is either higher than the destination sequence number in the routing table, the sequence numbers are equal, but the hop count plus one is smaller than the existing hop count in the routing table, or the sequence number is unknown. In addition, the source uses a Time To Live (TTL) count to limit the flooding of RREQ packets and control the overhead associated with the network. Finally, a HELLO message is broadcasted periodically to inform neighbor nodes about node existence. When an active node (node on the active route) detects a route failure (the neighbor node is unreachable; i.e., HELLO is not being received), it sends a route error (RERR) packet to the source node, which in turn, initiates a new RREQ.

Overhead is a major drawback of AODV because of the flooding of control messages (HELLO and RREQ packets). However, the overhead is low when compared to proactive routing protocols and it also needs less memory, on the other hand it only stores the routes on demand so, it is preferable in application to large scale networks.

\section{C) Intelligent Routing}

This current routing methodology uses AI methods based on biologically inspired paradigms in order to modify routing mechanism and reduce the number of forwarded RREQ (Bai, 2007).

\section{Ant-Inspired Routing Protocols}

The term intelligent comes from the behavior of swarm animals and insects such as bees and ants. The idea starts from when ants leave their ant-cell searching for food, they follow several routes for that task. Ants leave a chemical substance called pheromone when they are moving; the pheromone marks the route they are travelling and enable other ants to follow the same route. When an ant reaches an intersection, it has to make a decision which way to take next; the concentration of pheromone decreases due to diffusion which enables other ants to decide and select the newest route. (Pervasive and Enchev. 2011)

ARA (Ant-Colony-Based Routing Algorithm) (Gunes et al., 2002) supports dynamic topology with multihop paths between nodes. Its method is based on individual ant and local information from them. Routing tables are not needed to be forwarded to other neighbor nodes.

Simple Ant Routing Algorithm (SARA) (Correia and Vazo, 2010) is a development to ARA. It offers low overhead facilitated through all three routing phases discovery, maintenance and recovery; this is done by means of three complementary mechanisms Controlled Neighbor Broadcast (CNB), maintaining active session's paths and deep search procedure that restricts the number of nodes searched.

ANSI (Ad hoc Networking with Swarm Intelligence) (Rajagopalan and Shen, 2006) is a unicast protocol for hybrid ad hoc networks that considers the existence of higher-capability mobile or stationary devices in a network. It is responsive to fluctuating topology and utilizes the common swarm intelligence routing strategies.

Ant Hoc Net (Di Caro et al., 2004) is an ant-based protocol using hybrid routing; it has reactive and proactive capabilities. HOPNET (Wang et al., 2009) is a hybrid protocol. It is based on ACO (ant colony optimization) and zone routing framework for broadcasting. HOPNET is highly scalable for large networks.

Intelligent MANet routing protocol system (IMAN) (Saeed et al., 2008) employs Genetic Algorithms to select the most optimum protocol based on the network context. It reduces packets delay and data load.

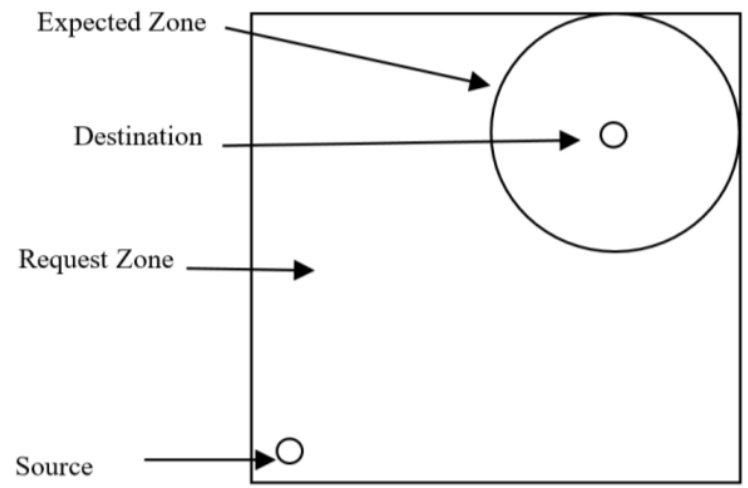

Fig. 2: LAR, scheme 1 
Verma et al., (2019) proposed two optimal routing, first routing technique is used to improve quality of service of AODV by using Mobile Software Agent (MSA) paradigm taking multiple parameters Received Signal strength, Remaining Energy, Queue length and delay together for finding path from source to destination node and second technique is based on fuzzy logic technique for finding optimal routing decision with four complex QoS matrices for MANET environment and capable to take optimal routing decision.

Cai et al. (2020) proposed an intelligent routing algorithm based on Prioritized Replay Double Deep QNetwork (PRD-DQN). two kinds of packet are designed: Fast Routing (FR) packet and Experience Transfer (ET) packet, in order to explore network and a reward function is defined in which congestion and channel quality are both considered for adaptive routing decision.

Sadreddini et al. (2021) proposed a new routing protocol by Implementing Intelligent Reflecting Surfaces (IRSs) in MANET, called MRIRS. MRIRS investigates the role of IRS as an intermediate node which helps not only for multi-flow multi-path routing even in Non-Line-of-Sight (NLoS) scenarios, but also supports delay minimization in the route discovery phase and helps with interference-avoiding channel assignment to the Primary Users (PUs).

\section{Algorithm, Discussion and Analysis}

The proposed routing protocol is based on LAR1 and ant's behavior. In best case scenario, source node has a record of the route to destination in advance because of previous connection to it, based on that it sends a RREQ to all neighbor nodes; the RREQ includes the expected path to destination. Only the nodes on the bath can resend the RREQ to their neighbors as well and so on until the RREQ reaches the destination which replies by RREP back to the source node. This way, the flood of RREQ is confined to the involved nodes in the route as shown in Fig. 3.

Reactive routing protocols such as AODV flood the whole network with the RREQ, position-based routing protocols, such as LAR1, flood confined area of the network with the RREQ, both cases consume more energy and cause network overhead. In contrast, Ant-OBS restricts the forwarding of RREQ just to the involved nodes, which in turn reduces the consumed power and the network overhead.

In another scenario where nodes are moving and the path from source to destination (source-A-D-GDestination) has changed (topology has changed) as shown in Fig. 3. Source node sends RREQ as normal including the previous path, RREQ reaches node $D$ based on the previous mechanism through node $\mathrm{A}$.

Now, node D forwards the RREQ to its neighbors A, $\mathrm{B}, \mathrm{C}, \mathrm{E}$ and $\mathrm{F}$, but none of them will send the RREQ because none of them are included in the path (the header of RREQ), node D acts as the new source, on behalf of the original source and waits for specific period of time to receive back RREP, when the time is up, node D floods the request zone with RREQ but the path, (ADGDestination), is not included, then nodes A, B, C, E and F, Neighbors of node $D$, will receive the new RREQ, but, only nodes $E$ and $F$ respond by RREP because they receive the same RREQ two times from the same neighbor and they are within the request zone as shown in Fig. 4. Then node $F$ finds node $G$, then node $G$ finds the destination as the traditional LAR1.

As noticed, the source node does not wait for certain time to resend the RREQ again and a node in the middle will act as the new source which confine the flood of RREQ and so reduces the network overhead and consumed power.

The following algorithm describes the previous explanation:

1. Source node send RREQ including the previous path used to contact the destination

2. If node is located on the path, node forwards the RREQ

3. Repeat step 2 until a node on the path does not receive RREP

4. The last node on the path that does not receive RREP from neighbor resends the RREQ, (the path is not included in the header of RREQ)

5. If the same RREQ received two times from the same neighbor AND located within the request zone, neighbor node resends the RREQ

6. RREQ is resent each time it is received by a node till it reaches the destination

7. Destination sends RREP back to the original source

The following figure; Fig. 5 illustrates the flowchart of sending RREQ by source node and receiving the RREP from destination in case of normal scenario.

\section{A) Mathematical Model}

Simulation representation is the common way to prove the validation of wireless routing protocols for MANET. This method depends on the accuracy and reliability of network simulator in addition to the impartiality of researcher in designing the scenario. On the other hand, mathematical verification is considered a crucial method for the evaluation of wireless routing protocols. The following presents the details of the mathematical model of the Ant-OBS protocol.

The degree of a node $N, d(N)$, is the number of nodes that are directly connected to $N$. A node is considered an isolated node if it has a zero degree (Cormen et al., 2001). 


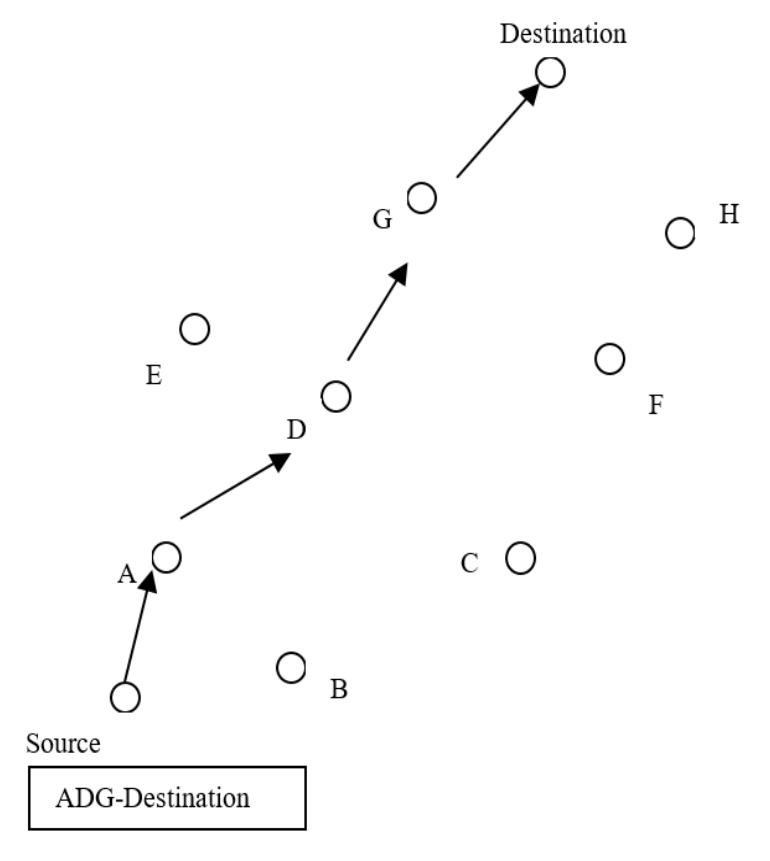

Fig. 3: Normal route

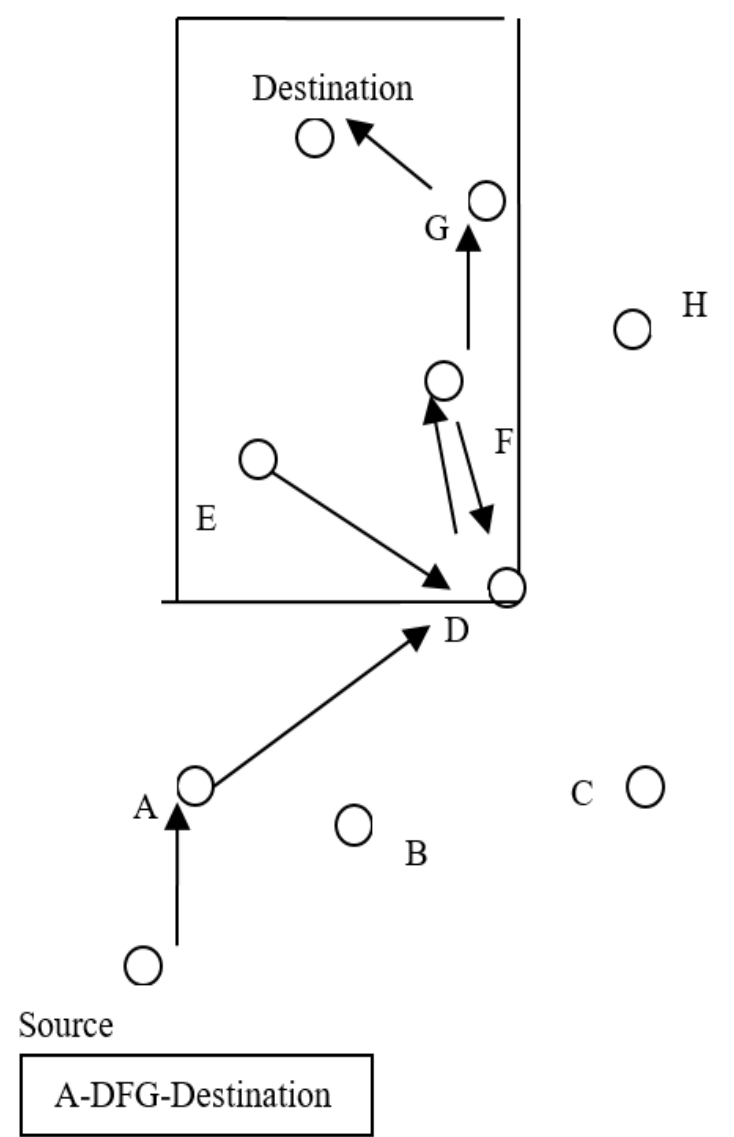

Fig. 4: Route discovered by Ant-OBS

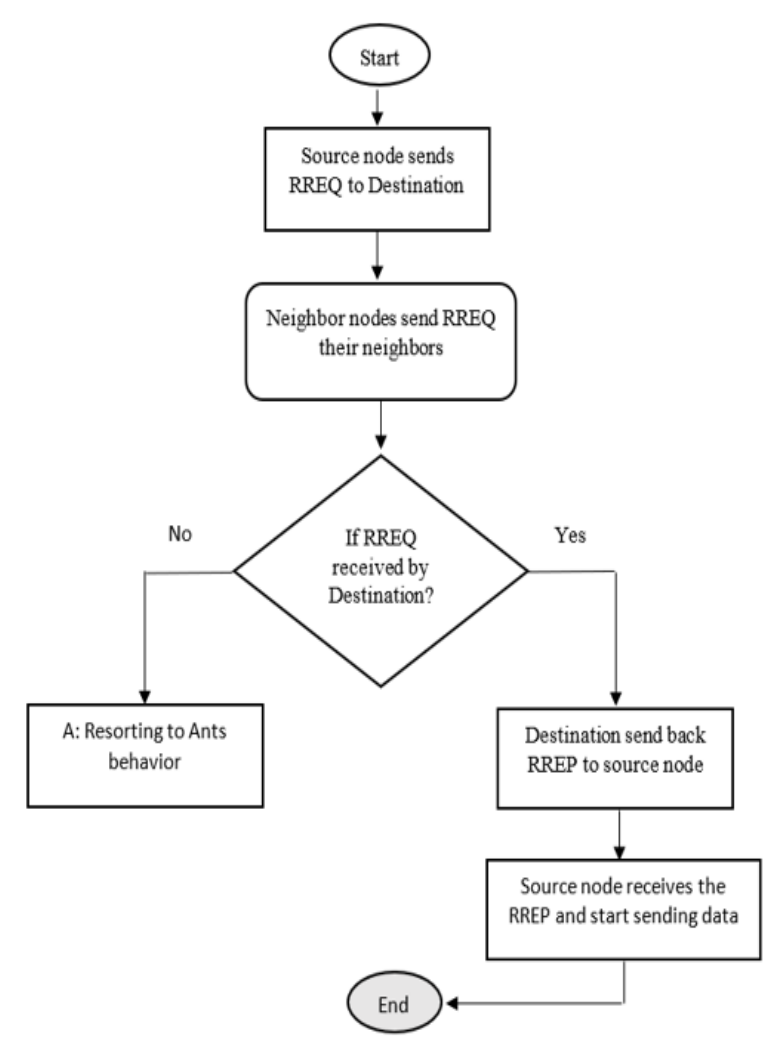

Fig. 5: Normal RREQ and RREP process

The probability $P$ that each node has at least $n_{0}$ neighbors is given by Eq. 1 (Bettstetter, 2002):

$P\left(d=n_{0}\right)=\frac{\left(\rho \mu r_{0}^{2}\right) n_{0}}{n_{0} !} \cdot e-\rho \mu r_{0}^{2}$

$n_{0}$ is the number of node's neighbors. $P$ is the probability that a randomly selected node has $n_{0}$ neighbors. $\rho$ is the homogeneous node density and $r_{0}$ is the transmission range of node.

The probability that a node will forward a RREQ to its neighbors successfully is broadcast forwarding probability (Saleem, et. al., 2010) is. RREQs may collide or get lost due to channel errors or dynamic nature of topology and so, broadcast forwarding probability is calculated using Eq. 2.

$p s=p r . p c \cdot p e$

$p s=$ The broadcast forwarding probability

$p r=$ The probability with which a node will forward a RREQ to its neighbors

$p r=$ The probability of not experiencing a collision at the MAC layer

$p e=$ The probability that the RREQ is not lost due to channel errors 
Notice that $p r$ is 1 in case of wireless routing protocols that flood RREQ over the whole network, $p r<1$ in case of position-based routing protocols because not all nodes are involved in forwarding the RREQ.

In this research, the network overhead is considered as the number of relayed RREQ packets, i.e., it is a function of Expected Forward Degree (Saleem et al., 2010).

When source node broadcasts a RREQ to its neighbors, the RREQ will be received on average by pc.pe. $d_{\text {avg }}$ nodes $\left(d_{\text {avg }}\right.$ is the average degree) and then each neighbor node receives the RREQ will forward it by probability $p r$. Then, the number of source's neighbors that will forward the received RREQ to their neighbors is $p s . d_{\text {avg }}$ nodes. Keeping accumulating the number of forwarding the received RREQ successfully each hop up to $h$ hops from the source (until RREQ is received by destination) represents the total number of network overhead which can be calculated using Eq. 3 (Jazyah, 2013):

$C p=\left\{\begin{array}{l}P_{s} d_{\text {avg }} \quad \text { if } h=1, \\ P_{s} d_{\text {avg }}+d_{\text {avg }} \sum_{i=1}^{h-1}\left(P_{s}\right)^{i+1} \prod_{j=1}^{i} d_{f}[j] \text { otherwise. }\end{array}\right.$

$C p$ is the total expected network overhead. $d f[j]$ is the expected forward degree of a node at $j$ hops. And $h$ is the number of hops from the source node.

It is concluded that the network route overhead is directly related to the number of hops traversed by a RREQ, probability of forwarding a RREQ ( $p r)$ and the expected forward degree of nodes. When $p r=1$ (in the case of topology-based routing protocol such as AODV), RREQ is flooded over the whole network, the result is huge network overhead, when $p r<1$ (such as LAR1 and Ant-OBS), the Cp will be decreased in comparison to the previous case because of the restrictions that applied on selecting the next hop node, which leads to less pr which in turn leads to reduction in $\mathrm{Cp}$; this means that the total network overhead will be reduced as the case in the Ant-OBS protocol. As a result, it is an evidence that the network overhead of the Ant-OBS protocol is less than its counterparts - AODV and LAR1.

In addition, the least overhead protocol, i.e., the least number of nodes that forward the received RREQ, has the least consumed energy in transmission. In general, the network consumes less energy than the case when AODV and LAR1 are used.

The following are some results of the analytical model for the AODV, LAR1 and the Ant-OBS based on equation 3 (Network overhead).

Considering the following values: $P S=1$ in case of AODV with reference to equation 2 , if we consider a node will forward the RREQ successfully to 5 neighbor nodes, then Ps. davg $=5$ (the number of neighbors close to the source that will forward the received RREQ to their neighbors $)$ and $d_{\text {avg }}=5$ as $P s=1, d f[j]=3(d f[j]=(2 j+1) /(2 j$ $-1)$, Table 1 shows the values of parameters of Eq. 3 for AODV for three hops as an example.
Table 1: Routing overhead of AODV

\begin{tabular}{llllll}
\hline Ps & $\mathrm{d}_{\mathrm{avg}}$ & Ps.d $\mathrm{d}_{\mathrm{avg}}$ & $\mathrm{i}$ & $(\mathrm{Ps})_{\mathrm{i}+1}$ & $\mathrm{df}[\mathrm{j}]$ \\
\hline 1 & 5 & 5 & 1 & 1 & 3 \\
1 & 5 & 5 & 2 & 1 & $5 / 3$ \\
1 & 5 & 5 & 3 & 1 & $7 / 5$ \\
\hline
\end{tabular}

Substituting the values from Table 1 into equation 3 , then $\mathrm{Cp}(\mathrm{AODV})$ is 110

Using the same method to produce the table for LAR 1 protocol as shown in table 2 .

Table 2: Routing overhead of LAR1

\begin{tabular}{llllll}
\hline $\mathrm{P}_{\mathrm{s}}$ & $\mathrm{d}_{\text {avg }}$ & Ps.davg & $\mathrm{i}$ & $(\mathrm{Ps})^{\mathrm{i}+1}$ & $\mathrm{df}[\mathrm{j}]$ \\
\hline $3 / 5$ & 5 & 3 & 1 & $3 / 5$ & 3 \\
$3 / 5$ & 5 & 3 & 2 & $9 / 25$ & $5 / 3$ \\
$3 / 5$ & 5 & 3 & 3 & $27 / 125$ & $7 / 5$ \\
\hline
\end{tabular}

$\mathrm{Cp}(\mathrm{LAR} 1)$ is 44.16

Table 3: Routing overhead of AntOBS

\begin{tabular}{llllll}
\hline Ps & davg & Ps.davg & i & (Ps)i+1 & df[j] \\
\hline $1 / 5$ & 5 & 1 & 1 & $1 / 5$ & 3 \\
$1 / 5$ & 5 & 1 & 2 & $1 / 25$ & $5 / 3$ \\
$3 / 5$ & 5 & 5 & 3 & $27 / 125$ & $7 / 5$ \\
\hline
\end{tabular}

From the previous results, it is noticed that Ant-OBS behaves better in comparison to AODV (topology based) and LAR1 (position based). It has much less network overhead; AntOBS $<$ LAR $1<$ AODV

Next Table 4 summarizes the previous results for the three protocols

Table 4: Routing overhead's comparisons

\begin{tabular}{llll}
\hline & Ant-OBS & LAR1 & AODV \\
\hline $\mathrm{Cp}$ & 16.96 & 44.16 & 110 \\
\hline
\end{tabular}

using the same way to generate the table for Ant-OBS, but if we consider the total number of neighbor nodes that the source can send them the RREQ is 1 for two hops, then Ps $=1 / 5$, while the third hop (resort to LAR1 method) $\mathrm{Ps}=5$ as all node confined within the request zone participate in forwarding the RREQ, so Ps $=3 / 5$. Then the $\mathrm{Cp}$ (Ant-OBS) is 16.96 , Table 3 .

\section{Conclusion}

This work presents an algorithm for MANET that enhances the performance of network in terms of network overhead and power consumption. The proposed protocol (Ant-OBS) benefits from the behavior of ant insect when ant find intersection, it decides which route to follow, AntOBS selects the required route based on decision when no known next hop is predicted, it benefits from LAR1 in some stages to forward the RREQ.

The proposed protocol enhances the performance of wireless routing protocol by not forwarding the RREP back to the source; this way the source node will not initiate a new RREQ again because a node in the middle between source and destination will act as the source node; this will reduce the network overhead and power consumption of network as a whole. 
Most wireless routing protocols aim to reduce packet delay and load and some of them aim to select the best route based on pure AI techniques, but the proposed protocol, Ant-OBS, is based on position-based routing protocol and Ani based technique because of its simplicity and powerfulness that aims to reduce network overhead which reflects on the power consumption.

Mathematical model is designed that represents the network overhead. The model is applied to ADV, LAR1 and Ant-OBS, the results obtained from the model show that Ant-OBS outperform the previous protocol because of its technique in selecting next hop nodes.

\section{Acknowledgement}

We thank everybody who assisted us to improve this research.

\section{Ethics}

We testify that this research paper submitted to the Journal of Science Publication has not been published in whole or in part elsewhere.

This research project was conducted with full compliance of research ethics norms of Arab Open University - Kuwait.

\section{References}

Abolhasan, M., Wysocki, T., \& Dutkiewicz, E. (2004). A review of routing protocols for mobile ad hoc networks. Ad hoc networks, 2(1), 1-22. doi.org/10.1016/S1570-8705(03)00043-X

Bai, H. (2007). A survey on artificial intelligence for network routing problems. NM, USA: University of New Mexico.

https://www.semanticscholar.org/paper/A-Surveyon-Artificial-Intelligence-for-Network-

Bai/c5b9e102c4c058a84f5078d786f857b0df5123af

Beijar, N. (2002). Zone routing protocol (ZRP). Networking Laboratory, Helsinki University of Technology, Finland, 9, 1-12.

https://citeseerx.ist.psu.edu/viewdoc/download?doi= 10.1.1.19.5568\&rep=rep $1 \&$ type $=$ pdf

Bettstetter, C. (2002, June). On the minimum node degree and connectivity of a wireless multihop network. In Proceedings of the 3rd ACM international symposium on Mobile ad hoc networking \& computing (pp. 80-91). doi.org/10.1145/513800.513811

Cai, J., Wang, C., Lei, M., \& Zhao, M. J. (2020). An Intelligent Routing Algorithm Based on Prioritized Replay Double DQN for MANET. In 2020 IEEE 92nd Vehicular Technology Conference (VTC2020Fall) (pp. 1-5). IEEE. doi.org/10.1109/VTC2020Fall49728.2020.9348471
Cormen, T. H., Leiserson, C. E., Rivest, R. L., \& Stein, C. (2001). Introduction to algorithms second edition. The Knuth-Morris-Pratt Algorithm. https://cs.wmich.edu/gupta/teaching/cs6310/lecture Notes_cs6310/sorting\%20chapter\%202.pdf

Correia, F., \& Vazão, T. (2010). Simple ant routing algorithm strategies for a (Multipurpose) MANET model. Ad Hoc Networks, 8(8), 810-823. doi.org/10.1016/j.adhoc.2010.03.003

Di Caro, G., Ducatelle, F., \& Gambardella, L. M. (2004, September). AntHocNet: an ant-based hybrid routing algorithm for mobile ad hoc networks. In International Conference on Parallel Problem Solving from Nature (pp. 461-470). Springer, Berlin, Heidelberg. doi.org/10.1007/978-3-540-30217-9_47

Faychuk, V., Lavriv, O., Klymash, M., Zhebka, V., \& Shpur, O. (2019, July). Investigation of the Bellman-Ford Algorithm Enhanced for Remote Execution. In 2019 3rd International Conference on Advanced Information and Communications Technologies (AICT) (pp. 204-208). IEEE. doi.org/10.1109/AIACT.2019.8847727

Gunes, M., Sorges, U., \& Bouazizi, I. (2002, August). ARA-the ant-colony based routing algorithm for MANETs. In Proceedings. International Conference on Parallel Processing Workshop (pp. 79-85). IEEE. doi.org/10.1109/ICPPW.2002.1039715

Jazyah, Y. H. (2013). Analytic Model of MLAR1. COMMUNICATION SYSTEMS, 25.

http://universitypress.org.uk/library/2013/rhodes/EC S.pdf\#page $=25$

Jazyah, Y. H., \& Hope, M. (2010, March). A review of routing protocols for UWB MANETs. In International Conference on Computational Science and Its Applications (pp. 228-245). Springer, Berlin, Heidelberg. doi.org/10.1007/978-3-642-12179-1_21

Johnson, D. B., Maltz, D. A., \& Broch, J. (2001). DSR: The dynamic source routing protocol for multi-hop wireless ad hoc networks. Ad hoc networking, 5(1), 139-172. https://www.cse.iitb.ac.in/ mythili/teaching/cs653_s pring2014/references/dsr.pdf

Karp, B., \& Kung, H. T. (2000, August). GPSR: Greedy perimeter stateless routing for wireless networks. In Proceedings of the 6th annual international conference on Mobile computing and networking (pp. 243-254). doi.org/10.1145/345910.345953

Ko, Y. B., \& Vaidya, N. H. (2000). Location-Aided Routing (LAR) in mobile ad hoc networks. Wireless networks, 6(4), 307-321. https://link.springer.com/article/10.1023/A:10191 06118419

Kranakis, E., Singh, H., \& Urrutia, J. (1999). Compass routing on geometric networks. In in Proc. 11 th Canadian Conference on Computational Geometry. doi.org/10.1.1.42.9931 
Liao, W. H., Sheu, J. P., \& Tseng, Y. C. (2001). GRID: A fully location-aware routing protocol for mobile ad hoc networks. Telecommunication systems, 18(1), 37-60. https://link.springer.com/article/10.1023/A:1016735 301732

Macker, J. P., \& Corson, M. S. (1999). Mobile ad hoc networking and the IETF. ACM SIGMOBILE Mobile Computing and Communications Review, 3(1), 11-13. doi.org/10.1145/1321414.1321421

Manibushan, D. S., Ashmitha, L., \& Deena, A. (2016). Routing protocols for mobile ad hoc network based on swarm intelligence. International Journal of Latest Trends in Engineering and Technology Special Issue SACAIM 2016, pp. 71-77. e-ISSN-10: 2278-621X.

Mauve, M., Widmer, J., \& Hartenstein, H. (2001). A survey on position-based routing in mobile ad hoc networks. IEEE network, 15(6), 30-39. doi.org/10.1109/65.967595

Perkins, C., Belding-Royer, E., \& Das, S. (2003). RFC3561: Ad hoc on-demand distance vector (AODV) routing. doi.org/10.17487/RFC3561

Pervasive, I. E. (2011, June). MANET routing protocols based on swarm intelligence. In MSC SeminarPervasive and Artificial Intelligence Research Group, Department of Informatics, University of Fribourg.

Rajagopalan, S., \& Shen, C. C. (2006). ANSI: A swarm intelligence-based unicast routing protocol for hybrid ad hoc networks. Journal of Systems Architecture, 52(8-9), 485-504. doi.org/10.1016/j.sysarc.2006.02.006

Sadreddini, Z., Güler, E., Khalily, M., \& Yanikomeroglu, H. (2021). MRIRS: Mobile Ad Hoc Routing assisted with Intelligent Reflecting Surfaces. IEEE Transactions on Cognitive Communications and Networking. doi.org/10.1109/TCCN.2021.3084402
Saeed, N. H., Abbod, M. F., \& Al-Raweshidy, H. S. (2008, March). Intelligent MANET routing system. In 22nd International Conference on Advanced Information Networking and ApplicationsWorkshops (aina workshops 2008) (pp. 1260-1265). IEEE. doi.org/10.1109/WAINA.2008.286

Saleem, M., Khayam, S., \& Farooq, M. (2010). On performance modeling of ad hoc routing protocols. EURASIP Journal on Wireless Communications and Networking, 2010, 1-13. doi.org/10.1155/2010/373759

Ucan, E., Thompson, N., \& Gupta, I. (2007, November). A piggybacking approach to reduce overhead in sensor network gossiping. In Proceedings of the 2nd international workshop on Middleware for sensor networks (pp. 19-24). doi.org/10.1145/1376860.1376864

Verma, V. R., Sharma, D. P., \& Lamba, C. S. (2019, September). Improvement in QoS of MANET Routing by finding optimal route using Mobile Agent paradigm and Intelligent Routing Decision using Fuzzy Logic Approach. In 2019 International Conference on Computing, Power and Communication Technologies (GUCON) (pp. 725-730). IEEE. https://ieeexplore.ieee.org/abstract/document/8940459

Wang, J., Osagie, E., Thulasiraman, P., \& Thulasiram, R. K. (2009). HOPNET: A hybrid ant colony optimization routing algorithm for mobile ad hoc network. Ad Hoc Networks, 7(4), 690-705. doi.org/10.1016/j.adhoc.2008.06.001 